\title{
Impact of a 12-week water program on the respiratory function in breast cancer survivors
}

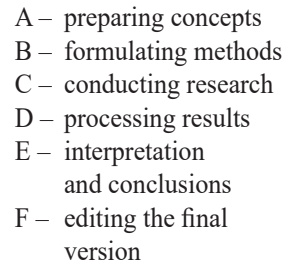

Tetiana Odynets ${ }^{1, A, D}$, Yuriy Briskin ${ }^{1, E-F}$, levgeniia Zakharina ${ }^{2, C, F}$, Anzhelika Yefremova ${ }^{3, B}$

${ }^{1}$ Department of Theory of Sport and Physical Culture, Lviv State University of Physical Culture

${ }^{2}$ Department of Theoretical Foundations of Physical and Adaptive Education, Classic Private University

${ }^{3}$ Ukrainian State University of Railway Transport

https://doi.org/10.5114/areh.2019.85018

\section{Abstract}

Introduction: The growing research demonstrates that breast cancer surgery and adjuvant radiation therapy greatly improve the long-term results of cancer treatment, overall survival, but leads to negative side-effects including from the respiratory system and quality of life. Aim: To evaluate the impact of a 12-week water program on the respiratory function in breast cancer survivors.

Material and methods: 68 patients who had survived breast cancer participated in a 12 -week physical rehabilitation. Pulmonary function parameters were evaluated in 34 women who performed water program (group A) and 34 women who received Pilates program (group B). Participants of both groups attended 36 rehabilitation sessions over 3 months.

Results: Based on the results of 12-week rehabilitation, it has been determined that proposed water program is more effective for improvement of pulmonary function in breast cancer survivors at outpatient rehabilitation. It was demonstrated that vital capacity and forced vital capacity were statistically higher by $270(\mathrm{p}<0.001)$ and $190 \mathrm{ml}$ $(\mathrm{p}<0.01)$, respectively in women of group A as compared to the group B. The respiratory minute volume was lower in group A by $1.06 \mathrm{l} / \mathrm{min}(\mathrm{p}>0.05)$ compared to the group B that indicated about more economical ventilation in rest and increasing the functional reserve of the respiratory system in breast cancer survivors.

Conclusions: The water-based program appears to be more effective then a Pilates program for improvements in vital capacity, forced vital capacity, maximal voluntary ventilation, and expiratory reserve volume in breast cancer survivors.

Key words:

women, water program, pulmonary function, breast cancer

\section{Introduction}

A growing body of research is demonstrating that a combination of surgery and adjuvant radiation therapy greatly improve the long-term results of breast cancer treatment, overall survival, but leads to negative side-effects including from the respiratory system and quality of life [1-3]. The literature suggests that cardiopulmonary toxicity, radiation pneumonitis, respiratory muscle weakness, impaired lung diffusion, activity-related dyspnea may occur in different periods of cancer treatment, as well as 
after its completion. The success of overcoming the negative effects of breast cancer treatment largely depends on the early start of rehabilitation activities and correct selection of special exercises [4,5].

A number of recent studies have shown positive effects of aerobic exercise, yoga and Pilates on the cardiopulmonary function in breast cancer survivors at outpatient rehabilitation [6-8]. For example the results of the study demonstrate that after eight weeks of regular training (3 times a week), there were significant changes in physical condition, endurance, decreases in fatigue, pain, depression and anxiety.

Pilates contributes to the development of chest and joint mobility, elasticity of ligaments, intermuscular and intramuscular coordination, strength endurance with minimal possibility of injury [6,8-10].

In addition, a few studies have shown the positive effect of performing physical exercises in the aquatic environment for improving the functional state of the respiratory system, reducing lymphostasis, and improving the psychoemotional state. This is facilitated by the hydrostatic pressure of water, the temperature factor and water resistance during the execution of motions [11-14].

Some papers have noted that breast cancer survivors often experience activity-related dyspnea, respiratory muscle weakness, deconditioning, exercise intolerance, and impaired lung diffusion $[6,8,12]$. According to recent studies [12-14], doing physical exercises is a key factor in improving muscular strength, endurance, cardiopulmonary function, and overcoming negative treatmentrelated side effects.

The primary outcome of this study was a pulmonary function assessment in breast cancer survivors at 12 weeks. As a consequence, the purpose of this research is to evaluate the impact of a 12-week water program as compared to a Pilates program on the respiratory function in breast cancer survivors.

\section{Materials and methods}

Sixty-eight women patients who had survived breast cancer participated in 12-week physical rehabilitation program. Women were randomized using sequentially numbered, opaque sealed envelopes. Pulmonary function parameters were assessed in 34 women who performed water program (group A) and 34 women who received
Pilates program (group B). Participants of both groups attended 36 rehabilitation sessions over 3 months. Informed consents were obtained from all the women included in the study. This research was approved by ethics Committee of Lviv State University of Physical Culture and performed according to the Helsinki Declaration principles.

Inclusion criteria were: participants after breast cancer surgery and in the 50-60 age range, I-II cancer stage, treatment-related lymphedema, written consent on paper, the period after surgery is from 2 to 6 months. Exclusion criteria were: presence of chronic nonspecific lung disease, congestive heart failure, III-IV cancer stage, period after surgery is more than 6 months.

Initially 75 potential participants were screened after breast cancer surgery for eligibility, 4 women of which were excluded for various reasons. The process of recruitment of women is presented at figure 1 .

The studied groups were homogeneous by the all demographic, treatment and pulmonary characteristics at baseline. Demographic and treatment-related characteristics of study participants are presented in Table 1.

Pulmonary function in breast cancer survivors was assessed by Spirometer SMP-21/01 RD (Monitor Ltd. Co., Rostov-on-Don, Russia). There were evaluated such parameters of pulmonary function in patients: vital capacity, forced vital capacity, forced expiratory volume in one second, respiratory minute volume, Mmximal voluntary ventilation, inspiratory reserve volume, and expiratory reserve volume. Current parameters were evaluated in raw data (litres or litres per second) and relative values ( $\%$ of predicted) that depended on age, weight and height of the woman.

Before performing spirometry, the equipment was calibrated and standard spirometry instruction was given to each patient. The women were tested in the seated and relaxed position wearing a nose clip with no air leaks between the mouth and the mouthpiece. Multiple maneuvers (i.e. vital capacity (VC), forced $\mathrm{VC}$, and maximal voluntary ventilation (MVV)) were obtained from each patient, and the spirometry values associated with the best maneuver were inputted into the database.

The following variables were assessed: (1) VC: the largest volume measured on complete exhalation after full inspiration, expressed in liters and was performed unforced. For VC, the largest value from 


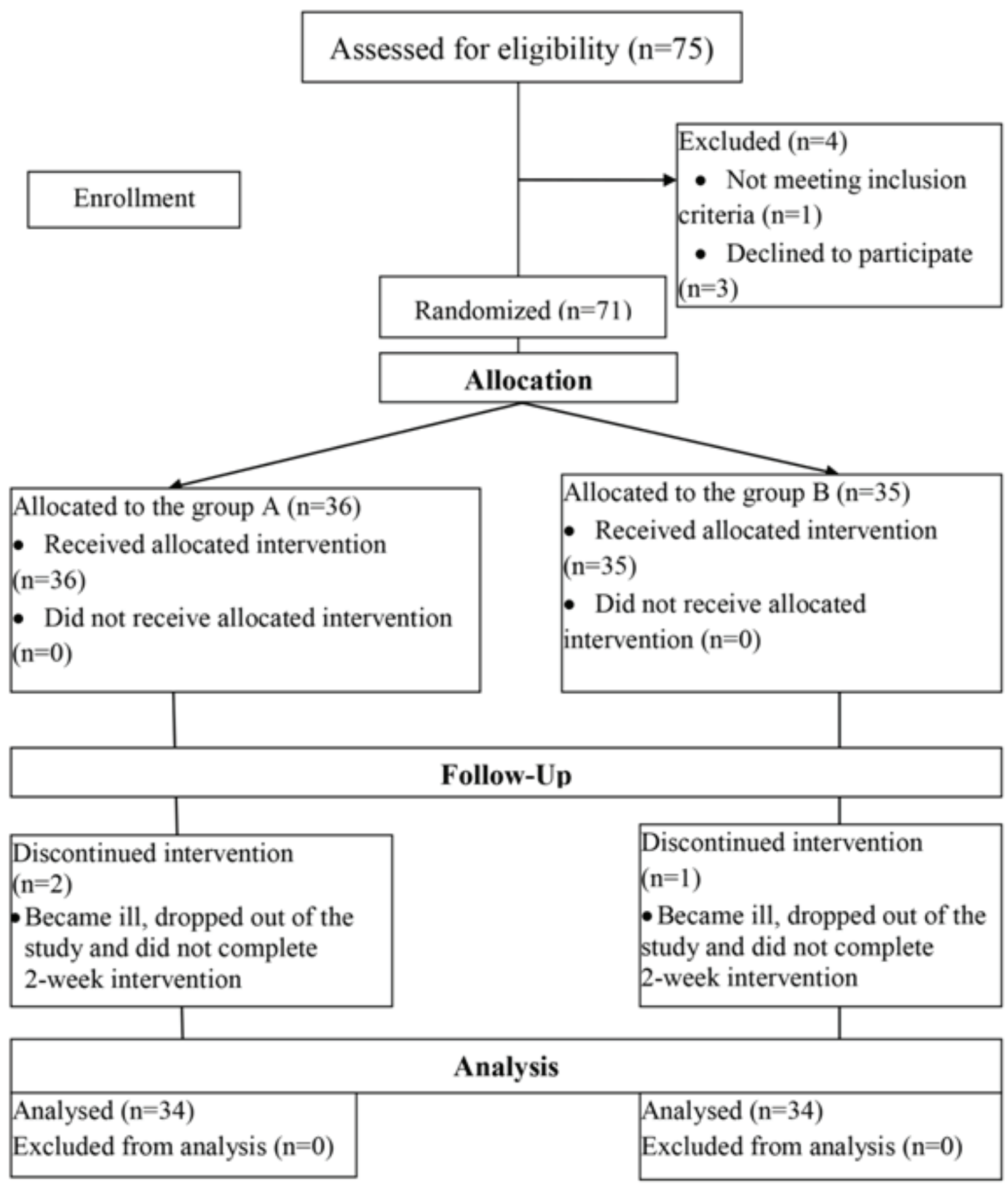

Figure 1. CONSORT flow diagram 
Tab. 1. Demographic and treatment-related characteristics of study participants

\begin{tabular}{|c|c|c|c|}
\hline Characteristics & $\begin{array}{l}\text { Water exercise group } \\
\qquad(\mathrm{n}=\mathbf{3 4})\end{array}$ & Pilates group $(n=34)$ & $\mathbf{P}$ \\
\hline Age, $(\mathrm{M} \pm \mathrm{m})$ & $57.44 \pm 2.16$ & $57.99 \pm 2.24$ & $>0.05$ \\
\hline \multicolumn{4}{|l|}{ Race: } \\
\hline White, n (\%) & $33(97 \%)$ & $32(94 \%)$ & $>0.05$ \\
\hline Black, n (\%) & $1(3 \%)$ & $2(6 \%)$ & $>0.05$ \\
\hline Married/committed relationship, $\mathrm{n}(\%)$ & $28(82 \%)$ & $27(79 \%)$ & $>0.05$ \\
\hline High school graduate, $\mathrm{n}(\%)$ & $13(38 \%)$ & $12(35 \%)$ & $>0.05$ \\
\hline College graduate, $\mathrm{n}(\%)$ & $19(56 \%)$ & $20(59 \%)$ & $>0.05$ \\
\hline Post-graduate, $\mathrm{n}(\%)$ & $2(6 \%)$ & $2(6 \%)$ & $>0.05$ \\
\hline Body mass index, $\mathrm{kg} / \mathrm{m}^{2},(\mathrm{M} \pm \mathrm{m})$ & $25.92 \pm 0.42$ & $26.01 \pm 0.81$ & $>0.05$ \\
\hline \multicolumn{4}{|l|}{ Treatment: } \\
\hline Surgery type (mastectomy by Madden), $\mathrm{n}(\%)$ & $34(100 \%)$ & $34(100 \%)$ & $>0.05$ \\
\hline \multicolumn{4}{|l|}{ Adjuvant therapy: } \\
\hline Radiotherapy & $30(88 \%)$ & $31(91 \%)$ & $>0.05$ \\
\hline Chemotherapy & $4(12 \%)$ & $3(9 \%)$ & $>0.05$ \\
\hline Time after surgery, months & $5.23 \pm 0.32$ & $5.11 \pm 0.42$ & $>0.05$ \\
\hline \multicolumn{4}{|l|}{ Cancer Stage: } \\
\hline Stage 1, n (\%) & $12(35 \%)$ & $13(38 \%)$ & $>0.05$ \\
\hline Stage $2, \mathrm{n}(\%)$ & $22(65 \%)$ & $21(62 \%)$ & $>0.05$ \\
\hline \multicolumn{4}{|l|}{ Degree of lymphedema: } \\
\hline $\mathrm{I}$-st degree, $\mathrm{n}(\%)$ & $5(15 \%)$ & $6(17 \%)$ & $>0.05$ \\
\hline II-nd degree, n (\%) & $19(56 \%)$ & $19(56 \%)$ & $>0.05$ \\
\hline III-rd degree, n (\%) & $10(29 \%)$ & $9(27 \%)$ & $>0.05$ \\
\hline
\end{tabular}

at least three acceptable maneuvers was recorded; (2) forced vital capacity (FVC): the maximal volume of air exhaled with maximally forced effort from a maximal inspiration, expressed in liters. The subject inhaled rapidly and completely from functional residual capacity while the breathing tube was inserted into the subject's mouth with lips sealed around the mouthpiece and tongue not occluding the mouthpiece; (3) forced expiratory volume in $1 \mathrm{~s}(\mathrm{FEV} 1)$ : the maximal volume of air exhaled in the first second of a forced expiration from a position of full inspiration, expressed in liters. FVC and FEV1 were measured from three forced expiratory curves that had an acceptable start of test and were free from artifact. The largest FVC and the largest FEV1 were recorded after examining the data from all of the usable curves; (5) MVV: the maximum volume of air a subject can breathe over a specified period (12 s), expressed in $1 / \mathrm{min}$. The subject was tested in the sitting position wearing a nose clip. After the subject makes an airtight seal around the mouthpiece, at least three resting tidal breaths should be obtained, followed by breathing as rapidly and deeply as possible; (6) inspiratory reserve volume (IRV): the maximal volume of air inhaled from end-inspiration, expressed in liters; and (7) expiratory reserve volume (ERV): the maximal volume of air exhaled from end-expiration, expressed in liters.

Obstructive disorders were identified only if: FEV1 $<80 \%$ predicted; and FEV1/FVC $<70 \%$. Restrictive disorders were identified only if: FEV1 was reduced $(<80 \%$ predicted normal); FVC reduced $(<80 \%$ predicted normal); and FEV1/FVC ratio normal $(>70 \%)$.

\section{Statistical analysis}

Parameters of pulmonary function were analyzed using Statistica for Windows software. To analyze the differences between studied groups, the independent samples t-test was used. To describe intra-group pre- and post-intervention changes dependent samples t-test was used. Spirometry parameters were presented as means and standard error of mean.

\section{Interventions}

The women of the group A carried out water program 3 times a week during twelve weeks. Participants of the group B performed Pilates program with the same frequency and duration. 
During the selection of appropriate means of water program and Pilates program to increase the pulmonary function in breast cancer survivors, one must observe the principle of adequacy of physical activity in accordance with the type of respiration disturbance and the level of functional state of cardiovascular system. The exercise programs were adapted to the individuals characteristics, and their general condition. The water program included: swimming, combined developing exercises, special exercises with noodles and swimming board, relaxation and strength exercises, regulated breathing exercises for the formation of an arbitrary breath hold on different phases of the respiratory cycle, considering the type of the respiration disturbance.

Static breathing exercises were used and aimed at improving pulmonary gas exchange, cardiopulmonary fitness, strengthening the respiratory muscles, reducing dyspnea, training for thoracic and diaphragmatic respiration. Dynamic breathing exercises were performed in conjunction with the limb movements and used to train the respiratory muscles, to increase the chest mobility, to normalize the metabolic processes, and to improve pulmonary ventilation.

The emphasis of the Pilates program included the diversity of breathing exercises, Hundreds, Spine stretch, Roll Down, scissors, Chester stretch, shoulder bridge, Dumb Waiter, Swan Dive, oneleg stretch exercises. Progressions of the Pilates program was made by increasing the complexity of the exercises, increasing their duration and intensity, including additional equipment.

\section{Results}

Repeated examination was conducted to assess the efficiency of the proposed rehabilitation programs for women after breast cancer surgery. Present study revealed a significant impact of the proposed water program and Pilates program on the pulmonary function in breast cancer survivors of both groups. Statistically significant improvements were identified in both groups $(\mathrm{p}<0.05)$.

Dynamics of respiratory function parameters in breast cancer patients during the intervention is presented in Table 2.

Tab. 2. Dynamics of respiratory function parameters in breast cancer patients during the intervention

\begin{tabular}{|c|c|c|c|c|c|c|c|}
\hline \multirow{2}{*}{\multicolumn{2}{|c|}{$\begin{array}{l}\text { Indicator } \\
\text { Beginning }\end{array}$}} & \multicolumn{2}{|c|}{ Group A $(n=34)$} & \multirow{3}{*}{$\begin{array}{c}\underset{\mathbf{p}}{\text { Beginning }} \\
<0.001\end{array}$} & \multicolumn{2}{|c|}{ Group B $(n=34)$} & \multirow{3}{*}{$\begin{array}{c}\mathbf{p} \\
>0.05\end{array}$} \\
\hline & & \multirow{2}{*}{$\begin{array}{c}\text { 12-week } \\
2.38 \pm 0,06\end{array}$} & \multirow[b]{2}{*}{$\begin{array}{c}2.81 \pm 0.04 \\
* *\end{array}$} & & \multirow{2}{*}{$\begin{array}{c}\text { 12-week } \\
2.41 \pm 0.04\end{array}$} & \multirow[b]{2}{*}{$2.54 \pm 0.05$} & \\
\hline Vital capacity, 1 & Actual & & & & & & \\
\hline & $\%$ of predicted & $76.03 \pm 2.52$ & $\begin{array}{c}89.50 \pm 1.82 \\
* *\end{array}$ & $<0.001$ & $75.93 \pm 1.54$ & $80.00 \pm 2.11$ & $>0.05$ \\
\hline \multirow[t]{2}{*}{ Forced vital capacity, 1} & Actual & $2.36 \pm 0.06$ & $\begin{array}{c}2.67 \pm 0.04 \\
* *\end{array}$ & $<0.001$ & $2.40 \pm 0.06$ & $2.48 \pm 0.04$ & $>0.05$ \\
\hline & $\%$ of predicted & $79.97 \pm 2.85$ & $\begin{array}{c}90.20 \pm 1.99 \\
* *\end{array}$ & $<0.001$ & $79.97 \pm 2.08$ & $82.57 \pm 1.94$ & $>0.05$ \\
\hline \multirow{2}{*}{$\begin{array}{l}\text { Forced expiratory } \\
\text { volume in } 1 \text { second, } 1\end{array}$} & Actual & $1.93 \pm 0.08$ & $2.23 \pm 0.05$ & $<0.01$ & $1.92 \pm 0.05$ & $2.22 \pm 0.03$ & $<0.001$ \\
\hline & $\%$ of predicted & $79.63 \pm 4.27$ & $91.37 \pm 2.46$ & $<0.01$ & $76.93 \pm 2.18$ & $89.27 \pm 1.93$ & $<0.001$ \\
\hline \multicolumn{2}{|c|}{ Respiratory minute volume, 1/min } & $7.17 \pm 0.60$ & $6.95 \pm 0.29$ & $>0.05$ & $7.51 \pm 0.34$ & $8.01 \pm 0.53$ & $>0.05$ \\
\hline \multicolumn{2}{|c|}{ Maximal voluntary ventilation, $1 / \mathrm{min}$} & $70.94 \pm 4.42$ & $\begin{array}{c}85.36 \pm 4.52 \\
* *\end{array}$ & $<0.01$ & $66.24 \pm 4.17$ & $67.88 \pm 4.02$ & $>0.05$ \\
\hline \multicolumn{2}{|c|}{ Expiratory reserve volume, 1} & $1.01 \pm 0.10$ & $\begin{array}{c}1.26 \pm 0.06 \\
* * *\end{array}$ & $<0.05$ & $0.87 \pm 0.05$ & $0.89 \pm 0.05$ & $>0.05$ \\
\hline \multicolumn{2}{|c|}{ Inspiratory reserve volume, 1} & $1.07 \pm 0.10$ & $1.17 \pm 0.05$ & $>0.05$ & $1.09 \pm 0.07$ & $1.20 \pm 0.06$ & $>0.05$ \\
\hline
\end{tabular}

Notes: ${ }^{* *}-\mathrm{p}<0.01 ;{ }^{* * *}-\mathrm{p}<0.001$ compared with the results of 12 -week intervention between group A and group B

Post-intervention results of spirometry indicators demonstrated a significant increase in vital capacity by $430 \mathrm{ml}(\mathrm{p}<0.001)$ in group A due to the predominant contribution in expiratory reserve volume, which significantly improved by $250 \mathrm{ml}(\mathrm{p}<0.05)$, and to a lesser extent by the inspiratory reserve volume, which increased by only $100 \mathrm{ml}(\mathrm{p}>0.05)$.
At the same time, the indicators of vital capacity, expiratory reserve volume and inspiratory reserve volume did not significantly change during the performing of Pilates program in patients of group $\mathrm{B}$, but their positive dynamics was noted by 130 , 110 and $20 \mathrm{ml}$, respectively ( $\mathrm{p}>0.05$ ). 
After the 12-week aquatic program, group A, we observed a significant increase in forced vital capacity by $310 \mathrm{ml}(\mathrm{p}<0.001)$, forced expiratory volume in one second - by $300 \mathrm{ml}(\mathrm{p}<0.01)$. In the patients of group B forced vital capacity did not changed, but forced expiratory volume in one second increased by $300 \mathrm{ml}(\mathrm{p}<0.001)$, which indicated about the strength raise of expiratory respiratory muscles and bronchial patency enhancement.

Post-intervention respiratory function analyses showed that respiratory minute volume slightly decreased in group A by $0.22 \mathrm{l} / \mathrm{min}$, that indicated a tendency to increase the functional reserve of the respiratory system, but in the group B it increased by $0.50 \mathrm{l} / \mathrm{min}$; these changes were not statistically significant.

Maximal voluntary ventilation increased by $14.42 \mathrm{l} / \mathrm{min}(\mathrm{p}<0.01)$ in group $\mathrm{A}$, in contrast to group B where it increased only by $1.64 \mathrm{l} / \mathrm{min}(\mathrm{p}>0.05)$. Obtained data indicated about improvements in the ability of patients to mobilize the available morphofunctional capabilities of the respiratory system, since this indicator can give you an idea about pulmonary function and the effectiveness of respiratory reserve.

Comparisons of the results of pulmonary function between the patients of group A and group $\mathrm{B}$ demonstrates a significant difference between these two groups after a 12 week rehabilitation intervention. It was indicated that vital capacity and forced vital capacity were statistically higher by $270(\mathrm{p}<0.001)$ and $190 \mathrm{ml}(\mathrm{p}<0.01)$, respectively in women of group A as compared to the group B. The respiratory minute volume was lower in group A by $1.06 \mathrm{l} / \mathrm{min}(\mathrm{p}>0.05)$ compared to the group B that indicated about more economical ventilation in rest and increasing the functional reserve of the respiratory system in breast cancer survivors.

\section{Discussion}

This study demonstrated that both programs facilitated improvements in the pulmonary function in breast cancer survivors. Nevertheless, after the 12-week physical rehabilitation intervention, the benefits of the water program were more impactful as compared to Pilates program.

Proposed water program applied differentiated exercises for respiratory system according to the type of the respiration disturbance. Intensity and duration of aquatic exercises applied in terms of the cardiovascular system of the patient. The results showed significant greater changes in most indicators of respiratory function in group A compared to group B.

We suggest that the positive impact of the water program was due to the fact that most of the exercises were clearly coordinated with the act of breathing. The exhalation occurred at the time of chest muscles contraction, and inhale - during the relaxation of arm muscles and relative expansion of the chest, which created optimal conditions for the functioning of the respiratory system.

Following on from the results of the 12-week physical rehabilitation, it has been established that water program helps to eliminate functional disorders of external respiration and increase its functional reserves.

A review of several studies [1-5] show that breast cancer survivors have functional and radiological pulmonary changes, that require increased attention from the physical therapist. Some studies emphasize the importance of water therapy [12-14] and Pilates program [8, 9] for improving cardiopulmonary fitness and overcoming upper extremity disorders in breast cancer survivors.

The results of our study support the prior findings [11-14] that a water program has positive effects not only for lymphedema reduction, improving psychoemotional state of women after surgical treatment, but also can lead to significant impact on vital capacity, forced vital capacity, forced expiratory volume in one second, maximal voluntary ventilation, inspiratory reserve volume, expiratory reserve volume.

A limitation of the study is that only our investigation was done on a limited number of patients and received results cannot be distributed to all breast cancer survivors.

In summary, we supported that proposed water program may be regarded as efficient method for the pulmonary function improvement in breast cancer survivors and was reasonable and safe. 


\section{References}

1. Blom U, Svane G, Anderson M, Wennberg B, Lind P. Long-term functional and radiological pulmonary changes after radiation therapy for breast cancer. Acta Oncol. 2014 Oct;53(10):1373-9. doi: 10.3109/0284186X.2014.934967.

2. Epler GR, Kelly EM. Systematic review of postradiotherapy bronchiolitis obliterans organizing pneumonia in women with breast cancer. Oncologist. 2014 Dec;19(12):1216-26. doi: 10.1634/theoncologist.2014-0041.

3. Odynets T, Briskin Y, Sydorko O. Psycho-emotional state and quality of life characteristics in women with post-mastectomy syndrome with different types of attitude to the disease. Physiotherapy Quarterly. 2018;26(1):9-12. doi: 10.5114/pq.2018.74706

4. O'Donnell DE, Webb KA, Langer D, Elbehairy AF, Neder JA, Dudgeon DJ. Respiratory Factors Contributing to Exercise Intolerance in Breast Cancer Survivors: A Case-Control Study. J Pain Symptom Manage. 2016 Jul;52(1):54-63. doi: 10.1016/j. jpainsymman.2016.01.004.

5. Kubo A, Osaki K, Kawanaka T, et al. Risk factors for radiation pneumonitis caused by whole breast irradiation following breast-conserving surgery. J Med Invest. 2009;56:99-110.

6. Eyigor S, Karapolat H, Yesil H, Uslu R, Durmaz B. Effects of pilates exercises on functional capacity, flexibility, fatigue, depression and quality of life in female breast cancer patients: a randomized controlled study. Eur J Phys Rehabil Med. 2010;46(4):481-7.

7. Odynets T, Briskin Y, Putrov S. Effectiveness of individualised intervention on pulmonary function in women with post-mastectomy syndrome. Physiother Practice Res. 2018;39(2):147-54. doi: 10.3233/PPR-180117

8. Stan DL, Rausch SM, Sundt K, Cheville AL, Youdas JW, Krause DA, Boughey JC et al. Pilates for breast cancer survivors. Clin J Oncol Nurs. 2012;16(2):131-41. doi: 10.1188/12.CJON.131-41.

9. Şener HÖ, Malkoç M, Ergin G, Karadibak D, Yavuzşen T. Effects of Clinical Pilates Exercises on Patients Developing Lymphedema after Breast Cancer Treatment: A Randomized Clinical Trial. J Breast Health. 2017;13(1):16-22. doi: 10.5152/ tjbh.2016.3136.

10. Kayıran O, De La Cruz C, Tane K, Soran A. Lymphedema: From diagnosis to treatment. Turk J Surg. 2017;33(2):51-7. doi: 10.5152/turkjsurg.2017.3870.

11. Ambroza C, Geigle PR. Aquatic exercise as a management tool for breast cancer-related lymphedema. Topics Geriatr Rehab. 2010;26:120-27.

12. Lindquist H, Enblom A, Dunberger G, Nyberg T, Bergmark K. Water exercise compared to land exercise or standard care in female cancer survivors with secondary lymphedema. Lymphology. 2015 Jun;48(2):64-79.

13. Ergin G, Karadibak D, Sener HO, Gurpinar B. Effects of Aqua-Lymphatic Therapy on Lower Extremity Lymphedema: A Randomized Controlled Study. Lymphat Res Biol. 2017 Sep;15(3):284-91. doi: 10.1089/lrb.2017.0017.

14. Tidhar D, Katz-Leurer M. Aqua lymphatic therapy in women who suffer from breast cancer treatment-related lymphedema: a randomized controlled study. Support Care Cancer. 2010;18(3):383-92. doi: 10.1007/s00520-009-0669-4. 